\title{
EFFECTS OF A FOURTH FAMILY ON $b \rightarrow s \gamma$ AND A USEFUL PARAMETRIZATION OF QUARK MIXING FOR RARE B DECAYS
}

\author{
Wei-Shu HOU \\ Department of Physics and Astronomy, University of Pittsburgh, Pittsburgh, PA 15260, USA
}

\author{
A. SONI \\ Department of Physics, University of California, Los Angeles, CA 90024, USA
}

and

\author{
Herbert STEGER \\ Department of Physics, University of Michigan, Ann Arbor, MI 48109, USA
}

Received 30 January 1987; revised manuscript received 7 April 1987

\begin{abstract}
The effects of a fourth family on the decays $b \rightarrow s(d) \gamma$ are reported. The $B R(b \rightarrow s \gamma)$ can become $\sim 10^{-3}$, i.e., a gain from the three-generation result by an order of magnitude. For very massive $t^{\prime}\left(m_{l}>200 \mathrm{GeV}\right)$ the four-generation result is not sensitive to $m_{1}$, making the reaction usuful for probing the new mixing angles of the fourth generation. We emphasize that combined information from $b \rightarrow 5 \gamma, s \ell^{+} \ell^{-}$and the hadronic $b \rightarrow s$ transition without charmed particles would be useful for constraining mass scales and mixing parameters of a fourth generation. In view of the importance of $B$ decays we present a new parametrization of the $4 \times 4$ mixing matrix that appears to be better suited for the study of $B$ physics.
\end{abstract}

The importance of the studies of rare B decays cannot be overemphasized. Just like studies of rare kaon decays, loop-induced transitions provide a probe of heavy quark physics. But rare B decays are even better in several ways. The B meson is ten times heavier than the kaon. Its lifetime is prolonged even more than the kaon's (relatively speaking) due to the smallness of the Kobayashi-Maskawa (KM) mixing matrix [1] element $V_{\mathrm{cb}}$ and $V_{\mathrm{ub}}$. But more important, loop effects involve the element $V_{\mathrm{ts}}^{*} V_{\mathrm{tb}}$ which is much larger than the kaon counterpart $\left(V_{\mathrm{td}}^{*} V_{\mathrm{ts}}\right)$; in fact, rare B decays branching ratios (BRs) can be enhanced relative to the analogous $\mathrm{K}$ decay modes by a factor $\sim\left|V_{\mathrm{ts}}^{*} V_{\mathrm{tb}} / V_{\mathrm{cb}}\right|^{2}\left|V^{*}{ }_{\mathrm{td}} V_{\mathrm{ts}} / V_{\mathrm{us}}\right|^{-2} \sim 10^{5}$. If a fourth generation [2-5] exists, it is likely that the $\mathrm{B}$ system would also be a better probe of new parameters involving the fourth generation. In this letter we wish to discuss the effects of a fourth generation on the modes $b \rightarrow s(d) \gamma$ and suggest that these modes may be quite suitable for probing, in particular, the mixing angles. This paper complements the earlier work [6] which emphasizes that the modes $b \rightarrow s \ell^{+} \ell^{-}, s v \bar{v}$ are very interesting as the loop-induced $b \rightarrow s Z^{*}$ transition depends very sensitively [7] on heavy (virtual) quark masses. From the point of view of fourth-generation physics, another mode of interest that we have investigated [8] is the loop-induced transition $b \rightarrow s$ " $g$ " (where the gluon, $\mathrm{g}$, is not necessarily on its mass shell and the reaction materializes into charmless final states), which may get enhanced from the three-generation inclusive BR of o $1 \%$ ) to more than $10 \%$, provided of course the mixing angles are not suppressed. Taken together, these modes prompt us to discuss, in this paper, a new parametrization for the $4 \times 4 \mathrm{KM}$ matrix that seems to be better geared toward the study of $\mathrm{B}$ physics.

The induced $b \rightarrow s \gamma$ (the photon being on-shell) matrix element is [7]

$\mathscr{M}=\frac{e G_{\mathrm{F}}}{4 \sqrt{2} \pi^{2}}\left(v_{i} F_{2}^{i}\right)\left[\bar{s} \sigma_{\mu \nu} \varepsilon_{\mu} \mathrm{q}_{\nu}\left(m_{\mathrm{s}} L+m_{\mathrm{b}} R\right) b\right]$, 
where $v_{i} \equiv V_{\mathrm{is}}^{*} V_{i \mathrm{~b}}$ and $i$ is summed from 2 to $n$ (for $n$ generations) since the u-quark contribution is absorbed into the definition of $F_{2}^{i}$ through unitarity of the KM matrix. The form factor is

$$
\begin{aligned}
& F_{2}^{\prime}(x)=-Q\left[\left(-1 / 4 y+3 / 4 y^{2}+3 / 2 y^{3}\right) x-\left(3 x^{2} / 2 y^{4}\right) \log x\right] \\
& \quad+\left[\left(1 / 2 y+9 / 4 y^{2}+3 / 2 y^{3}\right) x-\left(3 x^{3} / 2 y^{4}\right) \log x\right]
\end{aligned}
$$

where $x=x_{i}=m_{i}^{2} / M_{\mathrm{W}}^{2}, y=y_{i}=x_{i}-1$, and $Q=2 / 3$ is the up-type intermediate quark charge. The width is

$\Gamma=\frac{\alpha G_{\mathrm{F}}^{2}}{128 \pi^{4}} m_{\mathrm{b}}^{5}\left|v_{i} F_{2}^{i}\right|^{2}$

where we have ignored the $\left(m_{\mathrm{s}} / m_{\mathrm{b}}\right)^{2}$ corrections. To reduce uncertainties, we use the ansatz

$\mathrm{BR}(\mathrm{b} \rightarrow \mathrm{s} \gamma)=\frac{\Gamma(\mathrm{b} \rightarrow \mathrm{s} \gamma)}{\Gamma(\mathrm{b} \rightarrow(\mathrm{u}, \mathrm{c}) \ell \mathrm{v})} \cdot \mathrm{BR}(\mathrm{B} \rightarrow \ell \bar{v}+x)=\frac{3 \alpha}{2 \pi} \frac{\left|v_{i} F_{2}^{i}\right|^{2}}{\left|V_{\mathrm{ub}}\right|^{2}+\left|V_{\mathrm{cb}}\right|^{2} f\left(m_{\mathrm{c}}^{2} / m_{b}^{2}\right)} \cdot 0.12$,

where $f(x)$ is the usual phase space correction factor. The three-generation result has been given in ref. [9] $\# 1$. For four generations, it would appear that we have too many parameters; fortunately, most of them are inessential [6]. First of all, u-, c-quark contributions are completely suppressed, because $F_{2}^{i}$ goes as $x_{i}=m_{i}^{2} / M_{\mathrm{W}}^{2}$ for small $x_{i}{ }^{\# 2}$. Therefore, $m_{\mathrm{i}}, m_{\mathrm{i}}$ enter as the prominent internal quark masses. As for angles, it was pointed out in ref. [6] that $\left|v_{\mathrm{u}}\right| \ll\left|v_{\mathrm{c}}\right| \lesssim 0.05$. Therefore, unitarity implies $v_{\mathrm{l}} \simeq-\left(v_{\mathrm{t}^{\prime}}+v_{\mathrm{c}}\right)$, i.e., $v_{\mathrm{t}} \equiv V^{*}{ }_{\mathrm{ts}} V_{\mathrm{tb}}$ can be expressed in terms of $v_{\mathrm{t}^{\prime}}$, if we take $v_{\mathrm{c}}(\simeq 0.05)$ as known. We thus plot $\mathrm{BR}(\mathrm{b} \rightarrow \mathrm{s} \gamma)$ versus $v_{1}$. (we will comment on the allowed ranges later), for the set of heavy quark masses $m_{1}=150,200,300 \mathrm{GeV}$, and $m_{\mathrm{t}}=50$ and $100 \mathrm{GeV}$.

The result (fig. 1) shows that the fourth generation may cause a significant increase (by more than one order of magnitude) from the expectations based on three generations. We note that for values of the relevant KM

\# Eq. (5) of ref. [9] is a factor of 4 too large, but the curves plotted are more or less correct for large $m_{\mathrm{t}}$.

$\$ 2$ The effects of including QCD corrections are discussed later.
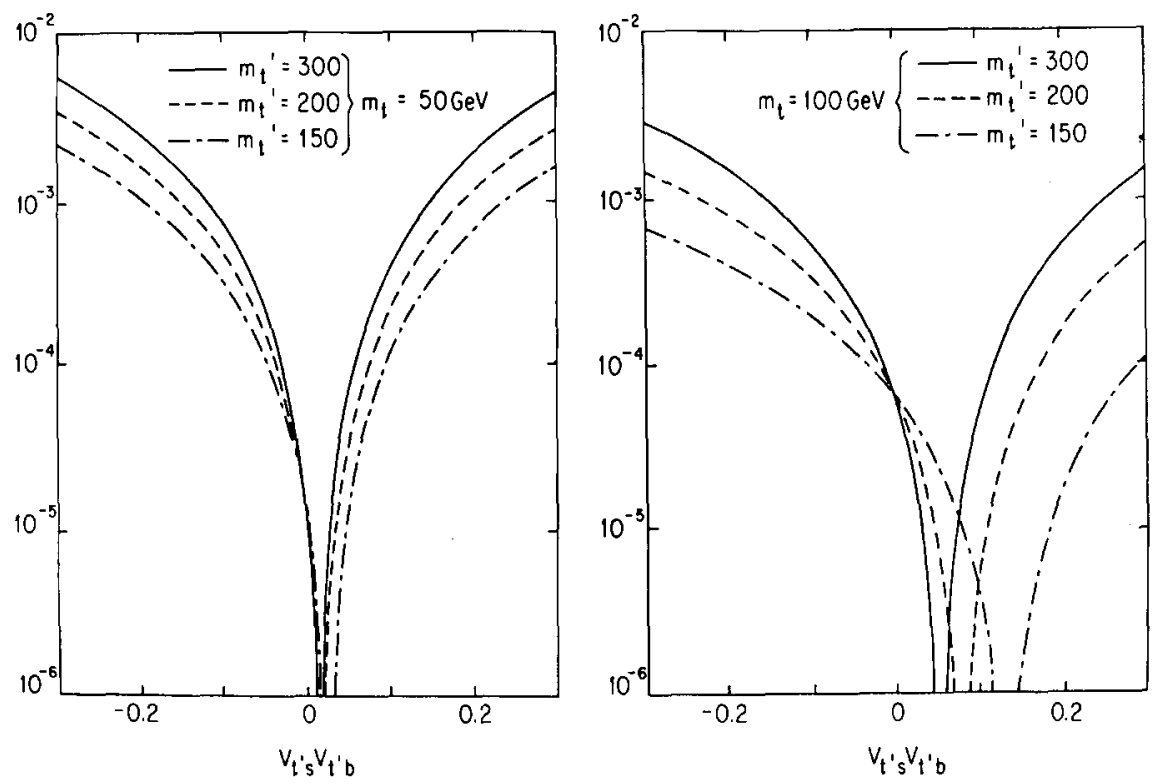

Fig. 1. BR $(\mathrm{b} \rightarrow$ sy $)$ versus $v_{1}$, for $m_{1}=150,200,300 \mathrm{GeV}$, and $m_{1}=$ (a) $50 \mathrm{GeV}$ and (b) $100 \mathrm{GeV}$. With QCD corrections taken into account, the results for $m_{1} \leqslant 100 \mathrm{GeV}$ should also look similar to (b). 
parameters to be of order of the Cabibbo angle, the BR can be of order $10^{-3}$. The current experimental limit $[10]$ is $\mathrm{BR}\left(\mathrm{B} \rightarrow \mathrm{K}^{*} \gamma\right) \leqslant 2 \times 10^{-3}$. Due to the usual uncertainties in evaluating hadronic matrix elements or questions of quark fragmentation, it is not clear (see discussion later) what this limit precisely implies for $b \rightarrow s \gamma$. If we take, as a guide, $\mathrm{BR}(\mathrm{b} \rightarrow \mathrm{s} \gamma) \sim 2 \mathrm{BR}\left(\mathrm{B} \rightarrow \mathrm{K}^{*} \gamma\right)$ [11] we see that the current experimental bound is not very informative about the fourth generation parameters. But if the experimental numbers improve by an order of magnitude to the $10^{-4}$ level, then, for $m_{1}=50 \mathrm{GeV}$ (i.e., fig. 1a) it seems that one may constrain $v_{\mathrm{t}}$, to within a narrow range $\left(-0.05 \lesssim v_{1} \lesssim 0.10\right)$ irrespective of $m_{1}$. This illustrates the potential utility of this mode (owing to its relative insensitivity to $m_{\mathrm{t}^{\prime}}$ ) in effectively constraining the four-generation mixing angle $V_{\mathrm{t}^{\prime} \mathrm{s}}^{*} V_{\mathrm{t}^{\prime} \mathrm{b}}$ similar to the way that the measurement of B life-time constrained $V_{\mathrm{cb}}$.

One can qualitatively understand the sensitivity of these curves to the mixing angle $v_{\mathrm{t}}$. From eq. (2) we see that for $x_{1}=m_{1}^{2} / M_{\mathrm{W}}^{2} \ll 1, F_{2}$ goes as $x_{1}$, whereas for large $x_{\mathrm{t}^{\prime}}, F_{2}$ varies roughly as $\log x_{\mathrm{t}}$. This result is to be contrasted with that for $\mathrm{b} \rightarrow \mathrm{s} \ell^{+} \ell^{-}[6]$. For the latter reaction the amplitude contains another form factor due to the induced $\mathrm{b} \rightarrow \mathrm{sZ}$ transition which, for $m_{\mathrm{t}} / M_{\mathrm{W}} \gg 1$ grows as $\left(m_{\mathrm{t}} / M_{\mathrm{W}}\right)^{2}$. Thus, as a consequence of suppression of top contribution and because the dependence on the heavy $t^{\prime}$ mass is mild the most sensitive fourthgeneration parameter dependence for $\mathrm{b} \rightarrow \mathrm{s} \gamma$ is on the mixing angle $v_{\mathrm{t}}$ rather than the mass $m_{\mathrm{t}}$.

The above discussion, however, also implies that this reaction $(b \rightarrow s \gamma)$ involving a real photon is unusually sensitive to QCD corrections (as has been pointed out recently [12]: QCD corrections could bring in simple logarithms of $x_{i}$ in addition to $F_{2}^{i}$ [13]. But for small $x_{i}$ a simple $\log$ would be much larger than $F_{2}$, and the loss due to the extra power(s) of $\alpha_{\mathrm{s}}$ can be more than overcompensated for by the appearance of these QCDinduced logs. This is more important for the contribution of a relatively light top quark, whereas for $t^{\prime}$ it is expected to be unimportant.

Thus the three-generation result gets appreciably modified (enhanced) for low $m_{\mathrm{t}}$. Since the $t$ and $\mathrm{t}^{\prime}$ contributions tend to cancel, the QCD corrections have the effect of making the process $(b \rightarrow s \gamma)$ less potent for the determination of $v_{1}$ than implied by fig. la. In fact, for $m_{1} \leqslant 100 \mathrm{GeV}$, the qualitative feature will look similar to the $m_{\mathrm{t}}=100 \mathrm{GeV}$ result shown in fig. $1 \mathrm{~b}$. Hence, for example, if $m_{\mathrm{t}}$, is not very heavy (less than 200 $\mathrm{GeV}$ say), the non-observation of $\mathrm{b} \rightarrow \mathrm{s} \gamma$ at the $10^{-4}$ level implies only $v_{\mathrm{t}^{\prime}} \gtrsim 0$, while for $m_{\mathrm{t}^{\prime}} \approx 200 \mathrm{GeV}$ one could infer that $0 \lesssim \nu_{1} \lessgtr 0.15$. Recall that had there been no QCD corrections, for $m_{\mathrm{t}}=50 \mathrm{GeV}$ we would have deduced from this BR limit the range $-0.05 \leqq v, \lesssim 0.1$ regardless of $m_{1^{\prime}}$. Thus, the reaction is not as effective and depends more on $m_{1}$; however, qualitatively, the point still remains.

Taking the relevancy of the $b \rightarrow s \gamma, b \rightarrow s \ell^{+} \ell^{-}, s v \bar{v}[6]$, and $b \rightarrow s^{\prime \prime} g$ " [8] processes into account, we ask the question that, in light of the possible existence of a fourth generation, what is the most practical parametrization of the $4 \times 4 \mathrm{KM}$ matrix for the near future? Historically, the discovery that $V_{\mathrm{ub}}, V_{\mathrm{cb}}$ are very small favored the Maiani form [14] of the $3 \times 3$ matrix over the others. Subsequently, it also became apparent to put [15] the single phase for the $3 \times 3$ case into $V_{\mathrm{ub}}$, since $C P$ would be conserved if $V_{\mathrm{ub}}$ vanishes. It was therefore not unnatural for various authors [2-5] to extend the Maiani form to the $4 \times 4$ case, particularly if they were concerned with $\epsilon,\left|\epsilon^{\prime} / \epsilon\right|$ and other kaon processes. (Some authors [16], taking cue from the apparent "angle hierarchy" of $\left|V_{\mathrm{us}}\right| \gg\left|V_{\mathrm{cb}}\right| \gg\left|V_{\mathrm{cb}}\right|$, have systematically generalized the Maiani-Chau-Keung (MCK) form to the $n \times n$ case.) However, if one wishes to study loop-induced rare B decays (or even B- $\bar{B}$ mixing), keeping the first row and fourth column simple while $V_{t^{\prime} s}, V_{t^{\prime} d}$ each become a sum of five terms (each term a product of sines and cosines) does not facilitate the discussion.

Now, direct experimental information on $V_{\mathrm{ub}}, V_{\mathrm{cb}}$, etc. clearly has to await the discovery of the fourth generation $b^{\prime}$ quark. One may have to rely on rare $B$ decays of the type suggested here and perhaps on $B-\bar{B}$ mixing as the only way that we can study the extra fourth-generation mixing parameters for quite some time to come. Secondly, there is no direct experimental evidence that $V_{\mathrm{ub}^{\prime}}, V_{\mathrm{cb}^{\prime}}$, etc. are small ${ }^{\# 3}$, and thus no evidence as yet

\#3 In fact, current experimental information on $V_{\mathrm{ud}}, V_{\mathrm{us}}, V_{\mathrm{cd}}, V_{\mathrm{cs}}, V_{\mathrm{ub}}, V_{\mathrm{cb}}$, etc., and unitarity of the $4 \times 4 \mathrm{KM}$ matrix implies only the limits $\left|V_{\mathrm{ut}}\right|,\left|V_{\mathrm{t}^{\prime} \mathrm{d}}\right| \leqq 0.1,\left|V_{\mathrm{cb}}\right|,\left|V_{\mathrm{t}^{\prime} \mathrm{s}}\right| \lesssim 0.7$ (see ref. [17] for more discussions), allowing considerable room, while $\left|V_{\mathrm{t}^{\prime} \mathrm{b}}\right|,\left|V_{\mathrm{tb}}\right|$ are practically unconstrained. 
that the angle hierarchy $\left|V_{\mathrm{cb}}\right| \ll\left|V_{\mathrm{cb}}\right|$, say, would continue. The studies of rare $\mathrm{B}$ decays will, therefore, be important for distinguishing between various possibilities. Thus a more pragmatic parametrization, geared towards the studies of rare B decays, is called for.

Fortunately, such a parametrization is possible. We want to have $V_{\mathrm{t}^{\prime} \mathrm{d}}, V_{\mathrm{t}^{\prime} \mathrm{s}}, V_{\mathrm{t}^{\prime} \mathrm{b}}$, etc. (elements of the fourth row) to be as simple as possible, viz. to avoid the clumsiness of having to deal with 3-5 separate terms. That is, we want to replace the simplicity of $V_{\mathrm{ub}}, V_{\mathrm{cb}^{\prime}}$, etc. (elements of the fourth column) in usual parametrizations of the $4 \times 4 \mathrm{KM}$ matrix, by the elements of the fourth row that are more relevant in the near future. At the same time, it would clearly be advantageous to retain a simple $3 \times 3$ limit (i.e., the MCK form), and also retain the simplicity of the third column for discussion of $b$ and $t$ decays. For a unitary matrix, it is always possible to choose to have one column and one row simple. We therefore choose to have the third column and fourth row simple and accordingly rearrange the sequence of multiplication of the various rotation matrices which we take from Botella and Chau [4]. Thus, from the limiting $3 \times 3$ MCK matrix [15]

$$
V_{3}=\left(\begin{array}{ccl}
c_{x} c_{z} & s_{x} c_{z} & s_{z} \exp \left(-\mathrm{i} \varphi_{1}\right) \\
-s_{x} c_{y}-c_{x} s_{y} s_{z} \exp \left(\mathrm{i} \varphi_{1}\right) & c_{x} c_{y}-s_{x} s_{y} s_{z} \exp \left(\mathrm{i} \varphi_{1}\right) & s_{y} c_{z} \\
s_{x} s_{y}-c_{x} c_{y} s_{z} \exp \left(\mathrm{i} \varphi_{1}\right) & -c_{x} s_{y}-s_{x} c_{y} s_{z} \exp \left(\mathrm{i} \varphi_{1}\right) & c_{y} c_{z}
\end{array}\right),
$$

we get the $4 \times 4$ matrix

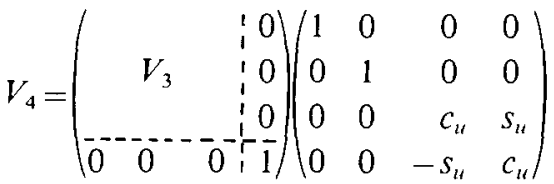

$$
\begin{aligned}
& \times\left(\begin{array}{ccll}
1 & 0 & 0 & 0 \\
0 & c_{v^{\prime}} & 0 & s_{\prime^{\prime}} \exp \left(-\mathrm{i} \varphi_{2}\right) \\
0 & 0 & 1 & 0 \\
0 & -s_{l^{\prime}} \exp \left(\mathrm{i} \varphi_{2}\right) & 0 & c_{v^{\prime}}
\end{array}\right)\left(\begin{array}{llll}
c_{w} & 0 & 0 & S_{w} \exp \left(-\mathrm{i} \varphi_{3}\right) \\
0 & 1 & 0 & 0 \\
0 & 0 & 1 & 0 \\
-S_{w^{\prime}} \exp \left(\mathrm{i} \varphi_{3}\right) & 0 & 0 & c_{w}
\end{array}\right)
\end{aligned}
$$

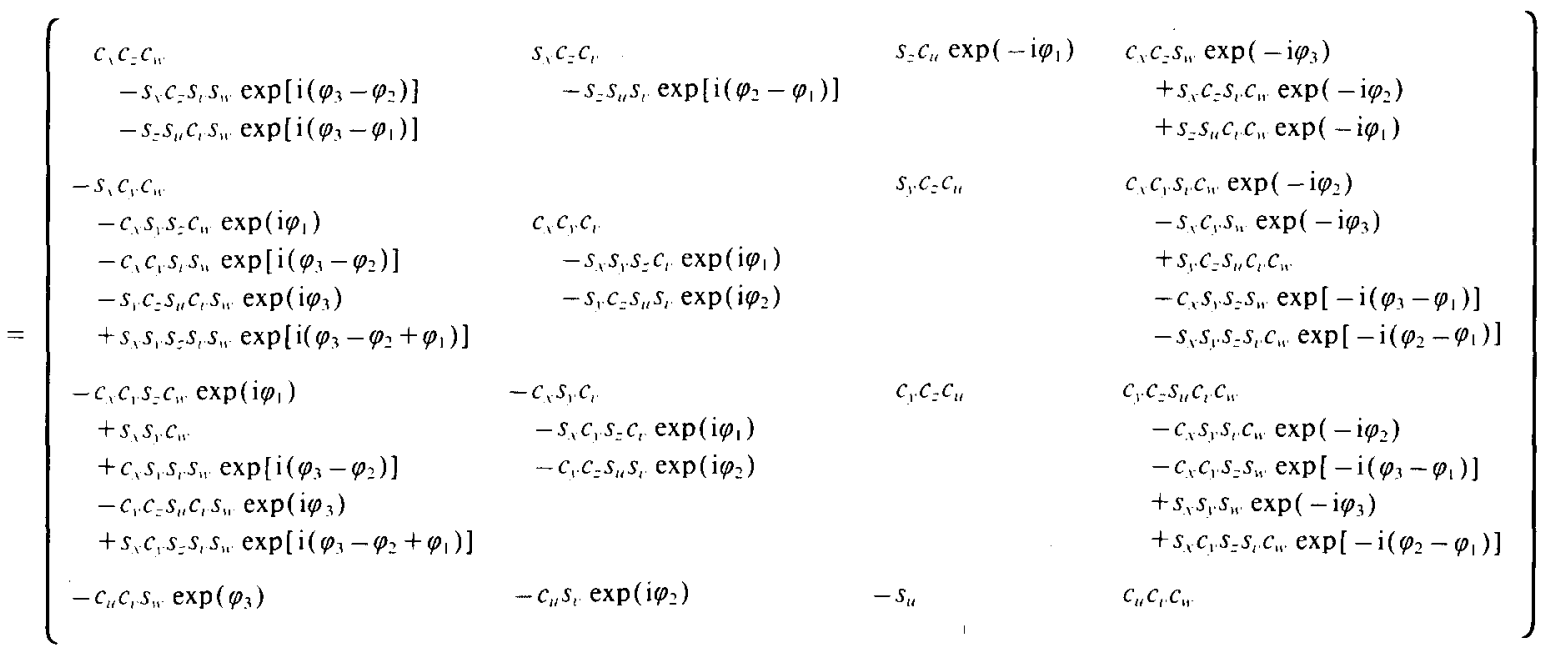

It is obvious that our parametrization is not optimized for studying kaon physics. However, our form is clearly more suitable for B physics, compared to the usual forms found in the literature. To elucidate this, imagine a possible scenario for the development of our understanding of the physics of heavy flavors in the 
next several years, as follows: Our knowledge of $V_{\mathrm{cb}}$ and $V_{\mathrm{ub}}$ should continue to improve through studies of $\tau_{\mathrm{B}}$, semi-leptonic B decay spectra, etc. The element $V_{\mathrm{tb}}$ may also become known, either through the measurement of $\Gamma_{\mathrm{t}}$ if the top quark is produced in $\mathrm{e}^{+} \mathrm{e}^{-}$machines, or by a detailed study of $\mathrm{W} \rightarrow \mathrm{tb}$ if $m_{\mathrm{t}} \leqslant M_{\mathrm{W}}$. Since in our parametrization of the $4 \times 4 \mathrm{KM}$ matrix the third column is kept simple, i.e., $\left|V_{\mathrm{ub}}\right|=s_{z} c_{u}$, $V_{\mathrm{cb}}=s_{y} c_{z} c_{u}, V_{\mathrm{tb}}=c_{y} c_{z} c_{u}$ and $V_{\mathrm{t} \cdot \mathrm{b}}=-s_{u}$, from the knowledge of $V_{\mathrm{ub}}, V_{\mathrm{cb}}, V_{\mathrm{tb}}$ we could infer $s_{z}, s_{y}, c_{u}$ and also predict $V_{\mathrm{t}^{\prime} \mathrm{b}}$. The other area of activity is the study of rare $\mathrm{B}$ decays, the ones advocated here, viz. $\mathrm{b} \rightarrow \mathrm{s} \gamma$, $b \rightarrow s e^{+} e^{-}, b \rightarrow s$ " $g$ ", etc. and also the study of $B_{d}-\bar{B}_{d}, B_{s}-\bar{B}_{s}$ mixing. These studies may lead to some knowledge of $V_{\mathrm{t}^{\prime} \mathrm{s}}^{*} V_{\mathrm{t}^{\prime} \mathrm{b}}$ and $V_{1^{\prime} \mathrm{d}}^{*} V_{\mathrm{t}^{\prime} \mathrm{b}}$ (and perhaps even $m_{\mathrm{t}^{\prime}}$ ). Since $\left|V_{\mathrm{t}^{\prime} \mathrm{s}}^{*} V_{\mathrm{t}^{\prime} \mathrm{b}}\right|=c_{u} s_{u} s_{v},\left|V_{\mathrm{t}^{\prime} \mathrm{d}}^{*} V_{\mathrm{t}^{\prime} \mathrm{b}}\right|=c_{u} s_{u} c_{u} s_{w}$, knowing $s_{u}$ through the studies of $V_{\mathrm{ub}}, V_{\mathrm{cb}}, V_{\mathrm{tb}}$ may allow us to set bounds on $s_{l}, s_{\mathrm{w}}$. Studies of $\mathrm{K} \rightarrow \pi v \bar{v}$ [5] may also lead to some knowledge of $V_{1 \mathrm{~d}}^{*} V_{\mathrm{t} \text { s }}$. Thus, because of the simplicity of the third column and fourth row, one can most easily derive bounds on the new mixing angles and thereby utilize those bounds to constrain $V_{\mathrm{td}}$, $V_{\mathrm{ts}}$ (which may also be studied when $\mathrm{t}$ is produced) and $V_{\mathrm{ub}}, V_{\mathrm{cb}}$ etc. For comparison, if one takes, e.g. the Botella-Chau parametrization [4], it would be difficult to do the same, since $V_{\mathrm{cb}}, V_{\mathrm{tb}}, V_{\mathrm{t} \text { 'd }}, V_{\mathrm{t}^{\prime} \mathrm{s}}$ are each composed of 3-5 terms. It is a matter of convenience, in the same spirit of choosing the MCK form for the $3 \times 3$ case, but of course the physics does not change. One may also subject the newly extracted fourth-generation angles $s_{u}, s_{t}, s_{w}$ to the constraints coming from the better known KM elements $V_{\text {ud }}, V_{\text {us }}$ and $V_{\text {cd }}$. However, no matter what parametrization one uses, it would be much harder to constrain the new phases.

Before closing, let us make several remarks on $b \rightarrow s \gamma$. Another practical difficulty in using the $b \rightarrow s \gamma$ mode for setting bounds $V_{l^{\prime} \mathrm{s}}^{*} V_{\mathrm{t}^{\mathrm{b}} \mathrm{b}}$ is the lack of knowledge of how much of $\mathrm{b} \rightarrow \mathrm{s} \gamma$ materializes as $\mathrm{B} \rightarrow \mathrm{K}^{*} \gamma$ (which is the only mode that has been studied experimentally). In the literature various authors have estimated this to be anywhere from $50[11]-98 \%[19]^{\# 4}$. Although it is easy to see that $\mathrm{B} \rightarrow \mathrm{K} \gamma$, it seems that the previous estimates may be high. The reason is that the $s$ quark is quite energetic and jet-like, and no calculational scheme seems to be trustworthy. However, the work of Grinstein, Wise and Isgur [20] on the decay $\mathrm{b} \rightarrow \mathrm{u} \ell \bar{v}$, though untrustworthy for the same reason does indicate that $\mathrm{B} \rightarrow \rho \ell \bar{v}$ constitutes a non-negligible fraction. Since $\mathrm{K}^{*}$ is heavier than $\rho$ and since $\mathrm{K} \gamma$ (and all the radial excitations of $\mathrm{K}$ ) is forbidden, it is plausible that perhaps $\mathrm{B} \rightarrow \mathrm{K}^{*} \gamma$ constitutes more than $20 \%$ of the $\mathrm{b} \rightarrow \mathrm{s} \gamma$ width. Radial excitations of $\mathrm{K}^{*}$ would seem to be important from the wave-function-overlap point of view (taking into account the large recoil momentum ) $\# 5$, but if one interpolated jet fragmentation down to this $2 \mathrm{GeV}$ "s-jet", it would seem unlikely that the $s$ quark materializes into a very massive object. As to non-resonant multiparticle production, from the fact that the average multiplicity for $\mathrm{e}^{+} \mathrm{e}^{-}$annihilation into hadrons at $5 \mathrm{GeV}$ is 4-5 [21], it seems that the $s$-quark jet would not fragment into many particles. We therefore think that $B \rightarrow K^{*} \gamma$ should be a reasonable, if not dominant, fraction of $b \rightarrow s \gamma$. In fact, this last point suggests that perhaps one should try to measure the inclusive BR for $b \rightarrow s \gamma$, by taking two- and three-prong (hadronic) events, calculate the invariant mass, and look for a recoiling photon with energy consistent with this mass; of course, triggering on hard photons $\left(E_{\gamma} \gtrsim 1 \mathrm{GeV}\right)$ is always an available alternative. We have no comment on how difficult the background subtraction in each situation is.

While this work was being completed, it came to our attention that the process $b \rightarrow s \gamma$ could be significant as a window to supersymmetric (SUSY) effects. The authors of ref. [22] point out that since (even after taking into account the large QCD corrections [12]) $\mathrm{b} \rightarrow$ s $\gamma$ is quite suppressed for low $m_{\mathrm{t}}\left(\leqslant M_{\mathrm{w}}\right.$ ) for the three-generation standard model, SUSY effects may dominate. Thus allowing one to set bounds on SUSY parameters from the present limit on $\mathrm{B} \rightarrow \mathrm{K}^{*} \gamma$. The reason is that the dominant SUSY contribution via a gluino-squark loop involves the strong rather than the weak coupling constant. This is an interesting observation. If $b \rightarrow s \gamma$ is observed at a rate inconsistent with that of a three-generation standard model $\left(\sim 10^{-4}\right.$

\footnotetext{
\#4 In deducing the $B R$ for $B \rightarrow K^{*} \gamma$, the recoil of the $K^{*}$ seems to have been ignored. We see no justification for this since the $K^{*}$ would be highly relativistic; of course, the use of non-relativistic wave-function-overlap formalism is also questionable.

${ }^{\# 5}$ We thank T. Altomari for discussions.
} 
for $m_{\mathrm{t}} \lesssim M_{\mathrm{w}}$ ), one would have to look for other signs of a fourth generation, such as through the processes $b \rightarrow s \ell^{+} \ell^{-}[6]$ and $b \rightarrow s^{s} g$ " [8] (which are less affected by SUSY), to distinguish between fourth generation or SUSY effects.

In summary, we point out ${ }^{\sharp 6}$ that $b \rightarrow s \gamma$ may be a useful probe of fourth-generation KM mixing parameters (a similar discussion holds for $b \rightarrow d \gamma$ ). In anticipation of the importance of rare $B$ decays we propose a new parametrization for the $4 \times 4 \mathrm{KM}$ matrix that we think is more pragmatic for studying $\mathrm{B}$ physics if there is a fourth generation. Progress in the studies of $b \rightarrow s \gamma, b \rightarrow s \ell^{+} \ell^{-}, b \rightarrow s^{\prime \prime} g$ ", top decay (if $t$ is discovered), B mixing, and perhaps $\mathrm{K} \rightarrow \pi v \bar{v}$ may even allow us to infer the bounds on new fourth-generation parameters.

We thank S. Bertolini, D. Cline, N. Deshpande, T. Ferguson, L.-F. Li, R. Willey and L. Wolfenstein for discussions. The work of WSH is supported by the NSF, and the works of AS and HS are supported by the DOE.

${ }^{2 h}$ After this work was completed, we received a paper by Hewett [23]. The approach and emphasis are somewhat different from ours.

\section{References}

[1] M. Kobayashi and T. Maskawa, Progr. Theor. Phys. 49 (1973) 652.

[2] See e.g. V. Barger, H. Baer, K. Hagiwara and R.J.N. Philips, Phys. Rev. D 30 (1984) 947.

[3] X.G. He and S. Pakvasa, Nucl. Phys. B 278 (1986) 905.

[4] F.J. Botella and L.-L. Chau, Phys. Lett. B 168 (1986) 97.

[5] U. Türke, Phys. Lett. B 168 (1986) 296, and references therein.

[6] W.S. Hou, R.S. Willey and A. Soni, preprint PITT-86-08 and UCLA/86/TEP/43, Phys. Rev. Lett., to be published.

[7] T. Inami and C.S. Lim, Progr. Theor. Phys. 65 (1981) 297, 1772(E).

[8] W.S. Hou, A. Soni and H. Steger, preprint PITT-87-02 and UCLA/87/TEP/2.

[9] B.A. Campbell and P.J. O’Donnell, Phys. Rev. D 25 (1982) 1989.

[10] P. Avery et al., Phys. Lett. B 183 (1987) 429.

[11] N.G. Deshpande and A. Soni, preprint UCLA/86/TEP/39, in: Snowmass '86, to be published.

[12] S. Bertolini, F. Borzumati and A. Masiero, preprint CMU-HEP 86-19; N.G. Deshpande, P. Lo, J. Trampetic, G. Eilam and P. Singer, preprint OITS 346.

[13] M.A. Shifman, A.I. Vainshtein and V.I. Zakharov, Phys. Rev. D 18 (1978) 2583.

[14] L. Maiani, in: Proc. 1977 Intern. Symp. on Lepton and photon interactions at high energies (DESY, Hamburg, 1977 ) p. 867.

[15] L. Wolfenstein, Phys. Rev. Lett. 51 (1984) 1945;

L.-L. Chau and W.-Y. Keung, Phys. Rev. Lett. 53 (1984) 1802.

[16] H. Harari and M. Leurer, preprint WIS-86/24-Ph; H. Fritzsch and J. Plankl, MPI-PAE/PTh 20/86.

[17] Particle Data Group, M. Aguilar-Benitez et al., Review of particle properties, Phys. Lett. B 170 (1986) 74.

[18] J. Ellis and J.S. Hagelin, Nucl. Phys. B 217 (1983) 189; G. Eilam, J.L. Hewett and T.G. Rizzo, preprint Technion-Phys-86-44.

[19] P.J. O'Donnell, Phys. Lett. B 175 (1986) 669.

[20] B. Grinstein, M.B. Wise and N. Isgur, Phys. Rev. Lett. 56 (1986) 298; preprint CALT-68-1311 and UTPT-85-37.

[21] T. Ferguson, private communication.

[22] S. Bertolini, F. Borzumati and A. Masiero, Phys. Lett. B 192 (1987) 437.

[23] J.L. Hewett, Radiative B decays with four generations, preprint DOE-ER 40200-091 and IS-J-2454. 